Meta

Journal des traducteurs

Translators' Journal

\title{
La traduction du vocabulaire anglais francisé dans l'œuvre de Jacques Ferron : une impossible épreuve de l'étranger
}

\section{Richard Patry}

Volume 46, numéro 3, septembre 2001

URI : https://id.erudit.org/iderudit/003797ar

DOI : https://doi.org/10.7202/003797ar

Aller au sommaire du numéro

Éditeur(s)

Les Presses de l'Université de Montréal

ISSN

0026-0452 (imprimé)

1492-1421 (numérique)

Découvrir la revue

Citer cet article

Patry, R. (2001). La traduction du vocabulaire anglais francisé dans l'œuvre de Jacques Ferron : une impossible épreuve de l'étranger. Meta, 46(3), 449-466. https://doi.org/10.7202/003797ar
Résumé de l'article

Cette étude porte sur les termes étrangers francisés dans l'œuvre de Jacques Ferron (ex. gagnestères pour gangsters ) et l'extrême difficulté que représente leur traduction en raison des propriétés langagières qui les caractérisent. Ces formes opaques nous amènent à observer, dans l'ensemble, des stratégies assez conservatrices de la part des traducteurs. Cette étude est également l'occasion de présenter des positions peu connues de l'auteur concernant la maîtrise des langues et la traduction de ses propres écrits. Une bibliographie des œuvres de Jacques Ferron traduites en anglais est présentée en annexe à cette étude. 


\title{
La traduction du vocabulaire anglais francisé dans l'œuvre de Jacques Ferron: une impossible épreuve de l'étranger
}

\author{
RICHARD PATRY \\ Université de Montréal, Montréal, Canada
}

\begin{abstract}
RÉSUMÉ
Cette étude porte sur les termes étrangers francisés dans l'œuvre de Jacques Ferron (ex. gagnestères pour gangsters) et l'extrême difficulté que représente leur traduction en raison des propriétés langagières qui les caractérisent. Ces formes opaques nous amènent à observer, dans l'ensemble, des stratégies assez conservatrices de la part des traducteurs. Cette étude est également l'occasion de présenter des positions peu connues de l'auteur concernant la maîtrise des langues et la traduction de ses propres écrits. Une bibliographie des œuvres de Jacques Ferron traduites en anglais est présentée en annexe à cette étude.
\end{abstract}

\section{ABSTRACT}

This study is concerned with the phenomena of gallicized foreign words in the works of Jacques Ferron (e.g. gagnestères for gangsters), and especially with the resistance they raise in terms of translatability, due mainly to their particular linguistic properties. We observe that the obscure nature of these words leads to a rather conservatice approach on the part of translators. This study also provided some original statements made by Ferron with regard to language mastering and the translation of his own works. Finally, a bibliography of Ferron's works translated into English is presented at the end of this study.

\section{MOTS-CLÉS/KEYWORDS}

difficulté de réexpression, Jacques Ferron, stratégies, termes étrangers francisés, traduction littéraire

J'ai l'impression que vous avez tort de vous en faire: toute écriture est traduction. Il faut être un peu malade pour s'y mettre, ou manquer de disposition pour la parole.

Jacques Ferron, Lettre à Julien Bigras ${ }^{1}$

\section{Introduction}

Jacques Ferron est souvent présenté comme une figure emblématique du questionnement collectif qui n'a cessé de s'affirmer depuis le début des années 1960 concernant le devenir du «pays incertain». Il l'a certes été. Tant dans ses œuvres de fiction que dans ses écrits polémiques ou dans ses activités militantes politiques et sociales, cet auteur a constamment cherché à esquisser les contours d'un Québec où la langue et la culture francophones puissent atteindre leur plein épanouissement.

Cependant, cette quête a eu pour conséquence inévitable un autre questionnement, parallèle, portant sur la nature de notre rapport à «l'Autre», et il est un second 
versant du personnage, encore relativement peu exploré à ce jour, que Ginette Michaud et Patrick Poirier ont récemment nommé «l'autre Ferron ${ }^{2} » ;$ celui qui s'intéresse aux aspects les plus divers, et parfois surprenants, de la vie du Canada anglais, des États-Unis et du Royaume-Uni.

Ce versant de «l'anglicité» est multiple et protéiforme dans l'œuvre de l'auteur, et peut parfois se réaliser sous la forme de personnages dans certains textes de fiction, comme par exemple, la caricature bonhomme et rieuse que représente Monsieur Northrop dans L'amélanchier ${ }^{3}$, la compassion aux profondeurs presque troublantes de Ann Higgit dans Les roses sauvages ${ }^{4}$ ou la flamboyante folie mégalomane de CDA Haffigan dans Le salut de l'Irlande ${ }^{5}$. Cette représentation peut également s'avérer plus nuancée et problématique. C'est le cas pour Frank Scott, qui apparaît sous différents noms dans plusieurs récits de Ferron, notamment dans Le ciel de Québec ${ }^{6}$, où il se nomme Frank (François) Anacharsis Scot, et où il reçoit l'insigne privilège d'être le narrateur du chapitre clôturant le récit. Son périple diégétique se termine cependant de façon abrupte dans Les confitures de coings ${ }^{7}$ où, sous le nom de Frank Archibald Campbell, il est officiellement congédié de l'œuvre par son auteur dans un texte publié en appendice au récit ${ }^{8}$.

Cette question du rapport à «l'Autre» est aussi abordée par Jacques Ferron sous l'angle des principaux enjeux politiques qu'elle soulève, comme par exemple, la situation difficile de l'Acadie ${ }^{9}$, qui pose de façon brutale la question de la survie d'une communauté francophone minoritaire environnée de toutes parts d'une majorité anglophone, et celle non moins délicate du bilinguisme, inutile et encombrant pour la plus large part du Canada anglais, sournois et menaçant pour le Québec. La position de Jacques Ferron est on ne peut plus claire à ce sujet: le bilinguisme n'est possible sur un territoire donné qu'à condition que les langues en contact soient une langue véhiculaire et une langue de grande civilisation. Le contact du français et de l'anglais, deux langues d'une grande civilisation et possédant chacune une bibliothèque complète, ne peut, selon l'auteur, que mener à l'assimilation d'un groupe linguistique par l'autre ${ }^{10}$.

Puisqu'il est ici question de bibliothèque, mentionnons également les nombreuses références à la littérature anglo-saxonne contenues dans l'œuvre de Jacques Ferron. Il y a tout d'abord William Shakespeare, que Ferron considère comme le plus grand écrivain après Dieu (qui a écrit la Bible ${ }^{11}$ ), et Charles Dickens ainsi que Samuel Butler dont il semble particulièrement apprécier les œuvres ${ }^{12}$. Ce sont là les lectures anglaises que l'on cite le plus souvent comme étant celles de Ferron. Cependant, ce ne sont pas les seules, et l'auteur se montre également fort élogieux, entre autres, à l'endroit de James Joyce, Antoine Hamilton, George Eliot, Edgar Allan Poe, George Byron, D. H. Lawrence et John Milton. Il exprime cependant une opinion plus réservée à l'endroit de Jack Kerouac qu'il dit être d'une génération "anglifiée $e^{13}$ », et se montre d'une sévérité de jugement absolument implacable à l'endroit de Hugh MacLennan, dont il parle souvent comme d'un écrivain à la réputation surfaite: "Hugh MacLennan, auteur surestimé de "Two Solitudes", qui fait de l'acrobatie sur un grand zéro» (Escarmouches, vol. 1, p. 135).

Ce rapport à «l'Autre» peut enfin prendre des formes plus subtiles et créatives. C'est le cas notamment pour une singularité langagière particulièrement intéressante qui consiste en l'occurrence de formes lexicales principalement de langue anglaise dont la graphie a été francisée par l'auteur. Dans l'œuvre de Ferron, il y a donc des 
«gagnestères» [gangsters], du «ouiski» [whisky] et un «Tchiffe» [chief]. On y voyage $\mathrm{au}$ «Farouest» [Far West], en «Bici» [British Columbia], et à «Olivoude» [Hollywood]. On y rencontre des «Angliches» [English], «John Dé Groube» [John D. Grube] et «Maréchal Maclhouane» [Marshall McLuhan]. On s'y exprime également de façon énergique, en jugeant que certaines idées sont «ouenderfoules!» [wonderful!]. À certains faits qui se montrent contrariants on oppose un «néveurmagne!» [never mind!] retentissant, et quand on veut se faire menaçant, on s'exclame «ouatchâte!» [watch out!]. Enfin, on se pose également des questions. Lorsque l'on ne sait pas de qui il s'agit, on dit «hou?» [who?], et de certaines personnes que l'on cherche, on se demande «ouèredéare?» [where they are?].

C'est la manifestation de cette forme de «surconscience linguistique», pour employer l'expression fort heureuse proposée par Lise Gauvin ${ }^{14}$, qui retiendra notre attention pour l'essentiel de la suite de cette étude. Nous appuyant sur les résultats d'une recherche d'envergure réalisée en 1998, nous verrons tout d'abord quelle est l'ampleur du phénomène et sa distribution dans l'œuvre de l'auteur, pour présenter par la suite les principaux fondements langagiers et identitaires sur lesquels repose le fonctionnement de cette stratégie d'appropriation lexicale. Dans la deuxième partie de l'étude, nous examinerons les enjeux soulevés par ces formes particulières pour la traduction, et nous verrons enfin comment elles ont été intégrées aux traductions anglaises des œuvres de Jacques Ferron.

\section{Appropriation du lexique étranger dans l'œuvre de Jacques Ferron}

\section{Quantification et distribution}

L'étude de 1998 comportait quatre questions principales ${ }^{15}$. La première consistait à déterminer si le phénomène de francisation orthographique des mots étrangers est épisodique ou fréquent dans l'œuvre de l'auteur. Concernant cette question, un décompte effectué à partir d'une version électronique de l'œuvre publiée et de logiciels de recherche contextuelle a permis d'établir que cette œuvre contenait 348 occurrences et 76 vocables de ce type de lexique ${ }^{16}$. Ce résultat a donc permis d'établir qu'il s'agit d'un phénomène important sur le plan quantitatif, et qui ne se limite pas à quelques occurrences disséminées de façon éparse dans l'ensemble de l'œuvre.

La deuxième question consistait à établir si ce lexique étranger démontrait une quelconque prédilection en termes de catégorie grammaticale. Cette question nous a paru importante sur le plan de l'analyse du langage en raison de la portée stylistique du phénomène ici en cause. Les résultats obtenus ont permis d'établir que des 76 vocables relevés dans l'œuvre de Ferron, 33 (43,42\%) sont des noms propres (de personnes, de lieux, d'institutions et de corporations ou marques commerciales), $30(39,47 \%)$ sont des noms communs ou des adjectifs qualificatifs, $11(14,47 \%)$ sont des interjections ou des expressions diverses, et 2 (2,64\%) seulement sont des verbes.

La troisième question consistait à déterminer si ce lexique étranger était uniquement de langue anglaise, qui tant en raison du passé colonial du Québec, que de son histoire récente ou de sa position actuelle en Amérique du Nord, est encore aujourd'hui la seule «autre» langue en compétition véritable avec le français. Les résultats démontrent donc de façon prévisible qu'une très forte proportion (71 vocables, $93,42 \%)$ du vocabulaire ici concerné est de langue anglaise. Cependant, 5 vocables sont 
d'une origine autre. Ce sont trois noms propres d'origine germanique: Loutiquenne [Lutiken], Shouadzeur [Schweitzer] et Quéclin [Koechlin]; un nom de ville danois: Elséneur [Elseneur/Helsingør] ${ }^{17}$; et un nom propre d'origine grecque: le Démon Stène [Démosthène].

La dernière question portait sur la distribution globale des occurrences dans les quatre genres textuels qui forment l'œuvre publiée de l'auteur: récit, polémique, théâtre et relations épistolaires ${ }^{18}$. Les résultats obtenus ont entre autres permis d'établir que le genre du récit regroupait à lui seul près de $70 \%$ du total des occurrences (241 occurrences, $69,25 \%{ }^{19}$ ) alors que la proportion pour les trois autres genres se répartit de la façon suivante: textes polémiques (14,95\%), pièces de théâtre $(13,22 \%)$ et relations épistolaires (2,58\%). Enfin, le résultat le plus marquant dans l'analyse de cette quatrième question consiste en le fait que près de $45 \%$ du total des occurrences dénombrées pour le genre du récit sont concentrées dans le seul conte «La vache morte du canyon» (107 occurrences, 44,40\%), texte qui fait environ une trentaine de pages.

\section{Fondements langagiers et identitaires}

À son niveau le plus fondamental, ce processus de francisation consiste en l'expression simultanée d'un double mouvement d'appropriation et de mise à distance. Le fait d'utiliser, par exemple, la forme "gagnestères» pour dire [gangsters] signale en effet une volonté de l'auteur de rapprocher la graphie de ce terme anglais de celle du français, et donc de l'apparenter aux allures formelles de la famille lexicale francophone. Ce geste constitue donc une forme manifeste d'appropriation. Cependant, faute d'un ancrage effectif dans l'usage de la communauté - dont l'auteur est d'ailleurs fort conscient - , cette modification se limite à un exercice de travestissement rédactionnel et ne peut déboucher sur une véritable intégration. L'appropriation ne francise pas le terme et, d'une certaine façon, le maquillage de surface que constitue cette manipulation de la graphie signale encore plus l'étrangeté du mot aux yeux du lecteur que le terme anglais lui-même. Cet air de famille que lui confère une rapide francisation de surface n'est en fait qu'un masque de carnaval, tantôt rieur ou grimaçant, qui le situe dans un espace interlangagier vide et dépourvu d'historicité2 ${ }^{20}$. Les termes francisés de Jacques Ferron ont en effet pour caractéristique première de n'appartenir à aucune langue et de constituer un gommage ponctuel, un face à face, curieux et équivoque, de l'anglais et du français à l'intérieur du même mot.

Cette confrontation interlangagière qui se trouve au cœur du processus en fait d'emblée une forme d'expression à caractère identitaire. C'est la substance d'un certain «contentieux» avec "l'Autre» qui semble être, à la base, le moteur de cette forme de créativité orthographique. À ce titre, ces termes francisés sont opaques au même titre - mais peut-être pas de la même façon — que les segments modalisateurs exprimés en français. Dans le cas précis qui nous préoccupe, les termes francisés exercent sur le lecteur un renvoi forcé à leur ubiquité langagière avant que d'exercer une fonction référentielle. Leur mise en discours est d'abord la forme d'expression métalinguistique d'une désignation de l'autre langue, et ensuite la dénomination d'un quelconque segment de réalité.

Enfin comme dernière caractéristique principale, ces mots francisés comportent toujours une dimension axiologique, attribut qu'ils partagent avec l'ensemble des 
modalisateurs. Ces mots ne font pas que nommer au travers la fusion intralexicale de l'anglais et du français, mais ils posent également un jugement sur ce qui est nommé.

L'expression de cette forme de jugement de la part de l'auteur est complexe et n'est accessible au lecteur que par l'intermédiaire de la synthèse de trois sources d'informations principales.

Tout d'abord, la catégorie grammaticale du terme semble déterminer, dans une certaine mesure, la saillance, ou l'importance, de ce jugement de valeur. Un examen détaillé du corpus de termes francisés contenu dans l'œuvre de Ferron semble indiquer que les noms communs sont caractérisés par une forme de jugement de valeur assez atténuée ou floue, alors que ce jugement est, de façon générale, beaucoup plus affirmé et explicite lorsqu'il s'agit de noms propres (noms de lieux, de corporations, de marques commerciales), cette expression étant maximale lorsqu'il s'agit de noms de personnes ${ }^{21}$. Ensuite, la forme graphique de chaque terme, considérée pour ellemême, a également son importance. Le processus de francisation ici considéré ne se limite pas à un exercice de dénomination «bilangagier». Il comporte aussi une dimension proprement littéraire, surtout tangible dans la dimension ludique ou poétique qu'il met en jeu. Ainsi, le résultat concret d'un acte de francisation peut suggérer des analogies avec certains mots existant en français ou induire des connotations comme l'ironie, la moquerie, le ridicule. Par exemple «Nouillorque» pour [New York] ne peut manquer d'évoquer «la nouille» pour un locuteur francophone, et «Pirsonne» pour [(Lester B.) Pearson ${ }^{22}$ ] évoque le terme "personne», faisant référence à l'absence ou l'inexistence, choix qui n'est certes pas neutre au plan axiologique.

Enfin, le contexte énonciatif dans lequel se situe un terme francisé est également indispensable au lecteur dans la formulation d'une hypothèse fondée quant à ce qui est exprimé par l'auteur sur le plan axiologique. En effet, ni la catégorie grammaticale du terme, ni sa forme propre de francisation ne sont suffisantes, et les prédications qui sont rattachées au référent, les propriétés qui lui sont conférées jouent également un rôle déterminant dans le balisage de ce parcours interprétatif.

Selon la valeur spécifique que peut prendre chacune de ces trois variables, leur assemblage peut donner lieu à l'actualisation effective d'un nombre de cas de figures aussi nombreux que varié. Il n'est donc pas question dans la présente caractérisation d'adopter une approche réductrice, mais plutôt d'identifier ce que nous semblent être les principaux ressorts qui déterminent la mécanique de base d'un processus ouvert et créateur, opération dont les éléments les plus importants sont présentés dans la figure 1.

\section{Comment traduire les termes francisés de Jacques Ferron : questions et réponses}

\section{Jacques Ferron et la traduction}

Le Ferron de «l'anglicité», dont nous avons évoqué quelques aspects dans l'introduction de cette étude, a été perçu par les traducteurs bien avant les lecteurs critiques (francophones à tout le moins) ce qui explique sans doute en partie la fortune difficilement prévisible de l'œuvre de l'auteur en traduction anglaise: 9 ouvrages de fiction, 1 pièce de théâtre, plusieurs contes et textes polémiques dans une livraison spéciale de la revue Brick consacrée à l'auteur, et plusieurs textes épars dans diverses 
FIGURE 1

Principales composantes du processus de francisation du lexique étranger

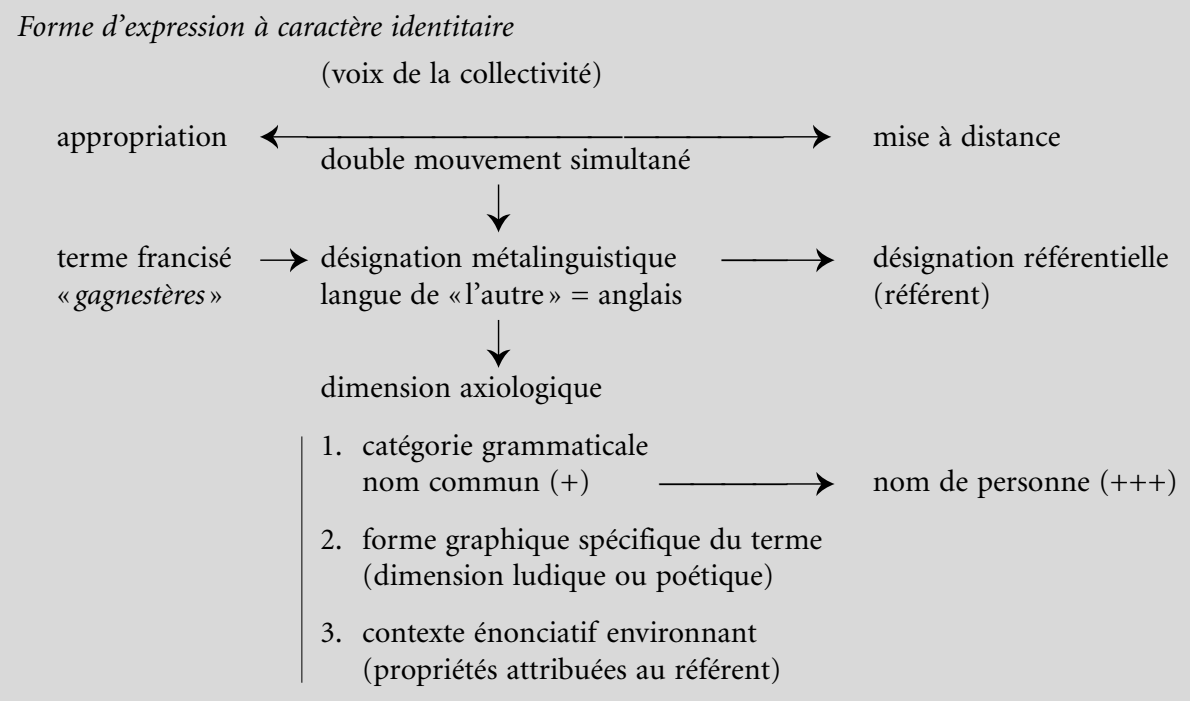

revues et anthologies ${ }^{23}$. Mentionnons également que plusieurs traducteurs ont contribué à ces diverses traductions, mais que seuls deux d'entre eux ont véritablement connu Jacques Ferron: Betty Bednarski, qui fut la première à traduire l'auteur, et avec qui il eut une profonde et véritable amitié ${ }^{24}$. Ensuite, Ray Ellenwood, avec qui il a entretenu une correspondance dont une partie a été publiée dans le collectif L'autre Ferron dirigé par Ginette Michaud et Patrick Poirier. Ajoutons enfin que selon les résultats d'une vaste enquête canadienne couvrant la période s'étendant entre 1965 et 1989, Jacques Ferron figure au huitième rang parmi les auteurs québécois francophones dont les œuvres ont suscité le plus d'études critiques au Canada anglais ${ }^{25}$.

Ce succès d'estime en traduction anglaise et au chapitre des études critiques anglo-canadiennes n'est pas négligeable pour un auteur québécois qui se définissait lui-même comme «mineur», et n'a pas été, concernant surtout les traductions, sans lui poser quelques dilemmes assez déchirants.

Tout d'abord, concernant son rapport à la langue cible de la traduction, Jacques Ferron fait énergiquement profession, tout au long de son œuvre, du fait de ne pas maîtriser la langue anglaise; à peine plus que son père qui ne s'exprimait en un anglais des plus rudimentaires qu'avec ses chevaux. Il s'agit là d'un constat étonnant pour un homme aussi instruit et cultivé qui, faut-il le rappeler, était d'abord et avant tout médecin, qui fut également un acteur des plus actifs sur les scènes politiques et sociales tant québécoise que canadienne, et qui passa un peu plus d'un an dans l'armée canadienne au sortir de ses études de médecine, engagement qui le conduisit à séjourner en Colombie-britannique, en Ontario, au Nouveau-Brunswick et enfin, à Grande-Ligne, au Québec. On a longtemps cru que l'auteur s'exprimait ainsi par boutade, mais en fait, selon la teneur d'une lettre adressée à Ray Ellenwood, il semble bien que ce n'était pas le cas. 
On a souvent cru que je ne parlais pas l'anglais par principe, mais que je le savais et pouvais éventuellement le parler si je le voulais. Je crois qu'il serait plus chic qu'il en soit ainsi, mais il n'en est rien. Je ne le parle tout simplement pas en dépit de toutes les occasions que j'ai eues de l'apprendre. (L'autre Ferron, p. 375)

Vient ensuite sa position très tranchée concernant le bilinguisme, que nous avons également soulignée dans l'introduction de cette étude, et selon laquelle cette situation de fait est possible dans un pays comme, par exemple, le Ghana, où l'éwé doit s'adjoindre l'anglais pour être fonctionnel, mais impossible entre deux langues de la même grande civilisation comme l'anglais et le français. Ce qu'il y a ici de particulièrement intéressant consiste en le fait que cette position est sous-tendue par une méfiance extrême, maintes fois exprimée par l'auteur, à l'égard des gens qui connaissent plusieurs langues.

[...] elle ressemble fort au goût que manifestent pour les langues ceux qui n'ont rien à dire. Ceux-ci semblent aussi nombreux que les ratés. On ambitionne de plus en plus d'être polyglotte sans doute en raison directe des facilités qu'on a pour le devenir. On fait grand cas de méthodes nouvelles. (Escarmouches, vol. 2, p. 172-173)

Cette méfiance n'était certes pas sans contaminer le rapport qu'entretenait Jacques Ferron avec la traduction. Bien qu'il n'ait exprimé aucune opinion portant de façon directe sur cette pratique, ses réserves sont cependant très perceptibles, par exemple, dans le portrait peu flatteur qu'il esquisse de Pierre Daviault, au moment de sa présidence de la Société des écrivains canadiens ${ }^{26}$.

Le président Daviault savait de quoi il parlait, ayant passé la majeure partie de sa vie à Ottawa, chanceux encore d'avoir conservé quelque esprit, peu il est vrai. Quant aux idées, je ne lui en ai pas connues. Et il était, en dehors des grammaires, des lexiques et des dictionnaires, d'une inculture rare. (Escarmouches, vol. 2, p. 41)

Enfin, il y a le fait de la traduction littéraire elle-même, dont l'auteur était conscient des écueils. Dans une lettre adressée à Ray Ellenwood, il soulève la question sans doute la plus fondamentale en rapport avec la traduction éventuelle d'un texte littéraire: pourquoi et pour qui écrit-on d'abord et avant tout?

Est-ce qu'on écrit pour être traduit, surtout quand on a commencé sans lecteurs. On écrit comme de bons enfants de Dieu, pour le bon Dieu lui-même, et l'on est tout surpris, un jour, d'apprendre qu'on a des lecteurs. Après arrivent les traducteurs, eux, ce sont des martiens, pas moins. (L'autre Ferron, p. 370).

Par des voies bien indirectes, ce passage semble nous ramener à la position exprimée par Walter Benjamin dans la première phrase de ses célèbres Prolégomènes ${ }^{27}$ : "Jamais, en présence d'une œuvre ou d'une forme d'art, la considération d'un récepteur ne s'avère fructueuse pour leur appréciation» (p. 13). Ayant ensuite rejeté l'utilité de faire appel à un «récepteur idéal», l'affirmation de Benjamin selon laquelle «il n'est besoin que de présupposer l'existence et l'essence de l'homme en général» (p. 13) ne serait-elle pas une autre façon de dire «le Dieu» de Ferron ${ }^{28}$ ? Et si l'auteur écrit pour Dieu, le traducteur littéraire, selon la caractérisation qu'en propose Pierre Deshusses $^{29}$, n'est souvent guère plus assuré dans sa tâche, et traduit sans trop savoir ce qui résultera de l'exercice « comme certains photographes photographient pour voir ce que cela va donner en image» (p. 49). Cependant, il se trouve d'autres rencontres sur la route de l'écrivain qui sont moins empreintes des délicatesses et des charmes 
de l'incertitude. Toujours dans sa correspondance avec Ray Ellenwood, Jacques Ferron évoque avec amertume la forme très dépersonnalisée dans laquelle a été réalisée la traduction de certaines de ses œuvres, expérience suffisamment traumatisante pour qu'il songe par la suite à se réserver le droit de ne pas être traduit dans ses contrats ultérieurs avec les maisons d'édition.

J'ai commencé Tchèque, fellow traveller, je pouvais comprendre. Ensuite, ce fut Madame Bednarski. Mes contes lui ont permis de se retrouver chez elle à Halifax à son retour de l'Ile de Wight ; c'était fortuit, je dirais même amical; il n'y avait pas de littérature dans ça. Après sont arrivés les autres, les vrais martiens, ceux que je ne connaissais pas et qui ne tenaient pas à me connaître, genre Harvesthouse, quelque chose comme ça, un genre que je n'aime pas et que l'éditeur m'impose. (L'autre Ferron, p. 370)

Enfin, il y a la délicate question de voir confronté ce qui a été «fait» par l'auteur à ce qui a été «refait» par le traducteur. À la différence des textes, que dans la foulée de la dénomination proposée par Jean Delisle ${ }^{30}$ on nomme maintenant «textes pragmatiques ", l'adéquation de la traduction du texte littéraire ne peut se mesurer à l'unique aune du transfert de sens, et pose des contraintes spécifiques qui sont complexes et nombreuses. En effet, une traduction juste du contenu langagier n'est pas nécessairement garante d'une bonne traduction littéraire, et les publications récentes dans le domaine s'entendent pour identifier trois formes de "nivellement» ou de "déformation» qui doivent absolument être évitées dans la traduction littéraire. Tout d'abord le nivellement culturel, qui consiste à déposséder le texte de certaines des caractéristiques de la culture native du texte d'origine, et qui a pour conséquence une traduction ethnocentrique qui fait taire l'une des nombreuses formes que peut emprunter la voix de l'étranger ${ }^{31}$. Ensuite, le nivellement stylistique, qui gomme le relief, les aspérités proprement langagières du texte d'origine, comme par exemple le phénomène lexical considéré dans cette étude, qui relève du niveau stylistique ${ }^{32}$. Enfin le nivellement idéologique qui consiste en des manipulations de contenu ou d'aspects formels du texte ayant pour but, conscient ou non, de le rapprocher des valeurs dominantes d'une société donnée ${ }^{33}$.

Sans disposer d'une représentation analytique aussi articulée et détaillée des difficultés posées par la traduction littéraire, Jacques Ferron en avait très bien saisi l'essence. Il était bien au fait de l'inépuisable valeur de cette monnaie d'échange qu'est le sens, tant dans le texte d'origine que dans son passage dans une autre langue. Il exprime d'ailleurs cette conscience dans un très beau passage d'une autre lettre adressée à Ray Ellenwood.

Je n'ai qu'un seul regret, celui d'être aussi cheval que mon père et de ne pouvoir traduire cet anglais en français pour que vous retraduisiez de nouveau; et l'on recommencerait souvent souvent, jusqu'à ce que les lettres soient complètement usées, disparues, et que nous soyons si fâchés l'un contre l'autre que nous ne voudrions pas nous serrer la main par le vide ainsi obtenu. (L'autre Ferron, p. 362)

\section{Et la traduction de Jacques Ferron}

Comment arriver à traduire les termes anglais francisés de Jacques Ferron? Comment rendre compte de ces traces d'opacité rédactionnelle de façon à les rendre à la fois accessibles au public lecteur de langue anglaise et à préserver les valeurs identitaires, métalinguistiques et axiologiques qui les caractérisent? Il s'agit là d'un 
défi qui est loin d'être simple, d'autant plus que la langue cible de la traduction est celle même de la confrontation intralexicale avec le français dans le texte d'origine.

L'ensemble des textes traduits de Jacques Ferron totalise 65 vocables et 231 occurrences de vocabulaire francisé. Nous verrons dans les développements qui suivent les trois stratégies principales utilisées par les traducteurs pour intégrer ces termes, sous une forme ou une autre, à leur traduction en langue anglaise.

\section{Intégration complète à la langue cible}

La première stratégie est certes la plus simple et consiste à rendre le mot anglais francisé du texte d'origine (ex. gagnestères) par le terme anglais correspondant (ex. gangsters). Celle-ci comporte le grand avantage de permettre de contourner un épineux problème de traduction, d'être simple et d'avoir pour conséquence une traduction systématique et cohérente. En contrepartie, elle constitue indéniablement une forme de nivellement stylistique assez brutale et vient gommer une source d'expressivité, une voix énonciative importante dans le texte d'origine.

Malgré ses désavantages notoires, cette stratégie est celle qui a été de très loin privilégiée dans l'ensemble des traductions et pour l'ensemble des traducteurs de Jacques Ferron. En effet, cette première stratégie a été appliquée à 45 vocables, soit $69,23 \%$ du total des effectifs.

Cette stratégie d'intégration peut d'abord prendre la forme d'une traduction directe du terme anglais francisé par son correspondant anglais ${ }^{34}$,

Pas un son de trop, juste le baragouin américain et les lamentations de suppliciés en coquetel parfait. (La charrette, p. 117)

No excess noise, just American jabber and the wail of dying prisoners in one perfect cocktail. (The Cart, p. 70)

ou d'une traduction de ce terme par un équivalent contextuel plus approprié:

«Bon, je lui dis, à cet O'Grady d'Archange: “Comme une jeune fille”. Autrement dit, rien de fort, de stréte ni d'amer, mais du sucré avec de l'alcool inaperçu» (Le ciel de Québec, p. 91)

"Right, so I says to this O'Grady of an archangel, I says, "Just like a sweet one thing", meaning nothing too strong, nothing neat or bitter, just nice and sugary to hide the alcohol» (The Penniless Redeemer, p. 74)

Mentionnons que ces intégrations complètes à la langue cible sont parfois accompagnées d'un renvoi à une note infrapaginale informant le lecteur de l'existence d'une opacité énonciative dans le texte source.

\section{Préservation de la forme francisée d'origine}

La seconde stratégie consiste à rendre le terme francisé du texte source (ex. gagnestères) en le conservant intact dans la traduction (ex. gagnestères). Par rapport à la précédente, cette seconde alternative comporte le même avantage de permettre de contourner un épineux problème de traduction, avec la différence, à première vue, de ne pas avoir pour conséquence fâcheuse le même résultat de nivellement stylistique. Dans une telle conjoncture, l'opacité énonciative de la prose ferronienne est en effet transportée dans la traduction, mais y conserve-t-elle cependant la même valeur? Le 
lecteur anglophone est-il en mesure d'apprécier les nombreux tours de passe- passe expressifs emmagasinés dans ces formes verbales ou ne risque-t-il pas plutôt d'être tout simplement dérouté par leur rencontre?

Il s'agit ici d'un pari délicat et périlleux qui a cependant été fait pour 18 vocables, représentant un peu plus du quart des effectifs $(27,69 \%)$. Ce pari a été fait par l'ensemble des traducteurs, qui accompagnent souvent ces termes de l'italique, d'une note infrapaginale, ou des deux.

Les vocables ici concernés ne forment cependant pas une distribution aléatoire. On peut dire pour les caractériser dans leur ensemble qu'il s'agit de ceux dont la force expressive est la plus grande, dont la valeur poétique atteint le sommet le plus élevé. Il s'agit, par exemple, des exclamations: "néveurmagne!», "ouennederfoule!» et «ouannedeurfoule ailledia!»; des questions que sont "hou?» et «ouèredéare?»; ensuite de quelques noms communs particulièrement évocateurs comme "Angliches», «cuiquelounche», "Farouest», "gagnestères», "mizeule» et «ouiski»; ainsi que de quelques noms propres comme «Andicotte», «CIPIAR» et «Ténesse».

La traduction du terme «Farouest» semble cependant avoir causé une certaine hésitation chez Julie May Stockton qui, dans la pièce de théâtre Les grands soleils, en a traduit deux occurrences par l'équivalent contextuel "out West», ce qui correspond à la stratégie d'intégration complète à la langue cible présentée dans le développement précédent:

Mithridate: Je parie qu'il va au Klendaque; il a l'air en retard. Mais il se rend peut-être aux States ou au Farouest. (Les grands soleils, p. 396)

Mithridate: I'll bet he's going up to the Klondike; seems a little late. But then again, he could be making his way the the States, or out West. (The Flowering suns, p. 78)

alors que pour la troisième, elle a opté pour la préservation de la forme telle qu'elle se présentait dans le texte d'origine, sans italique et sans note infrapaginale:

Mithridate: Le sachem, le sorcier, l'extravagance, les maringouins, les plumes et les totems, il mettait tout ça dans le Farouest, un peu en dessous du Klendaque.» (Les grands soleils, p. 650)

Mithridate: Indian chief, witch doctor, pagan ritual, mosquitos, feathers and totem poles, they all belong in the Farouest as far as he's concerned, somewhere just below the Klondike. (The flowering suns, p. 85)

\section{La solution du compromis: la trace de l'opacité énonciative}

La troisième possibilité qui s'offre au traducteur est une solution de compromis qui consiste à traduire le terme anglais francisé, mais de façon à ce qu'une trace de l'opacité énonciative du texte d'origine soit perceptible, et non par le recours à l'italique ou à une note infrapaginale, mais dans la forme traduite elle-même. Cette avenue est certes la plus difficile parmi celles évoquées dans cette étude. Elle présente cependant le grand avantage de représenter la forme optimale de traduction possible des termes francisés ferroniens qui permet une traduction de ces termes en langue anglaise, commode pour le lectorat, particulièrement lorsqu'il est unilingue, tout en évitant le grand désavantage de l'intégration complète, qui est un nivellement stylistique indésirable, le gommage d'une forme subtile d'expressivité du texte source. 
En raison des difficultés évidentes qu'elle pose, cette troisième possibilité est cependant peu populaire chez les traducteurs. Elle n'a en fait été utilisée que par Betty Bednarski, et dans l'unique traduction du conte La vache morte du canyon. Il s'agit cependant d'un texte particulier dont la caractéristique principale avait été soulignée dans un développement antérieur de cette étude: il contient à lui seul 12 vocables et 107 occurrences de termes anglais francisés, ce qui totalise 44,40\% des effectifs pour l'ensemble des textes de fiction.

En plus de la difficulté fondamentale à rendre compte de ces formes dans une traduction, ce texte pose donc à l'évidence un problème supplémentaire de taille: la justification d'une stratégie, quelle qu'elle soit, pour rendre compte d'un phénomène qui présente une si forte concentration en un seul texte. L'intégration complète entraînerait une perte inacceptable sur le plan de la discursivité. La préservation des formes d'origine risquerait de rendre le texte bizarre, ou tout simplement illisible pour le lectorat anglophone, et la solution du compromis, étendue à l'ensemble des occurrences, en plus de demander des prodiges d'imagination, risquerait de s'avérer une avenue presque aussi déconcertante que celle qui précède pour le lecteur. Donc, que faire?

Comme aucune solution d'ensemble n'était envisageable pour la totalité des effectifs, Betty Bednarski a utilisé une combinaison de deux des trois stratégies présentées dans cette étude pour sa traduction de La vache morte du canyon. Certains termes francisés ont donc été traduits par leurs équivalents respectifs anglais et 2 vocables $(3,08 \%)$ ont fait l'objet d'une traduction selon la stratégie du compromis ici discutée: «Farouest» et "touristeroume».

Betty Bednarski a traduit «Farouest» par «Farwest», écrit en un seul mot, plutôt qu'en deux mots séparés débutant chacun par des majuscules comme le veut l'orthographe d'usage anglaise (Far West). Elle a ensuite traduit le terme «touristeroume» par "Tourist Room» avec des majuscules au début de chaque mot lorsque le terme désigne l'établissement, et " [one of the] “tourist rooms" » avec des minuscules, mais entouré de guillemets, lorsqu'il désigne une chambre à l'intérieur de l'établissement.

La traductrice fait entre autres valoir comme argument le fait que ces adaptations orthographiques des mots anglais correspondent tout à fait au caractère purement rédactionnel des termes francisés de Ferron et qu'elles permettent de signaler ces fantaisies expressives de l'auteur tout en demeurant compréhensibles au lecteur anglophone. Cette argumentation est présentée dans deux études où la traductrice ${ }^{35}$ discute de façon détaillée de ses choix pour la traduction des termes francisés de Ferron en général, et plus particulièrement, de ses décisions dans la traduction de $L a$ vache morte du canyon.

Cette traduction de La vache morte du canyon a cependant fait l'objet de critiques, entre autres de Katy $\mathrm{Mezei}^{36}$. Celle-ci considère que le fait d'avoir fait disparaître toute trace de nombreux termes francisés a pour conséquence de priver le lecteur d'une importante partie de la symbolique de la dépossession contenue dans le texte d'origine (p. 390).

Cette critique n'est certes pas dépourvue de fondement. Cependant, il faut bien admettre que Betty Bednarski, seule traductrice d'ailleurs à avoir traduit ce conte, faisait face à un problème quasi insurmontable et lui donner le crédit qui lui revient d'avoir adopté une stratégie cohérente et systématique, de s'en être expliquée de fa- 
çon convaincante dans deux études — humilité dont peu des traducteurs de Ferron ont fait preuve $\mathrm{e}^{37}$ - et d'avoir produit une traduction qui est lisible et tout à fait acceptable, compte tenu de l'ingrate conjoncture langagière qui caractérise le texte source.

\section{En guise de conclusion}

Cette étude nous a tout d'abord permis d'explorer une facette particulière du Ferron de «l'anglicité»; dimension du personnage riche en substance, en nuances et parfois même en contradictions, dont l'essentiel reste à être "excavé» de l'œuvre dans les études critiques à venir. Et de façon plus particulière, cette analyse a également permis de mettre en évidence des positions peu connues exprimées par l'auteur: sa méfiance extrême à l'égard des gens qui maîtrisent plusieurs langues, et la prudente réserve dont il fait montre à l'égard de la traduction de ses écrits.

Enfin, la démonstration principale de cette étude concerne les termes anglais francisés de l'auteur et l'extrême difficulté que représente leur traduction en raison des caractéristiques identitaires, métalinguistiques et axiologiques dont ils sont porteurs. Ces formes opaques sont difficilement réexpressibles dans une autre langue, et nous avons été à même de constater qu'elles ont été la source de stratégies assez conservatrices de la part des traducteurs, si l'on fait exception de l'audace manifestée par Betty Bednarski dans sa traduction de «Farouest» et de «touristeroume» dans La vache morte du canyon.

En fait, il est des conjonctures purement langagières qui posent problème en traduction, que l'on contourne lorsque c'est possible, que l'on confronte quand on a moins le choix, et ce, avec un succès et une fortune relatives. Que l'on pense, par exemple, aux nombreuses propositions de titres français pour le roman Wuthering Heights d'Emily Brontë, pour lequel Les hauts de Hurle-vent, proposé par Frédéric Delebecque, s'est finalement imposé. Le problème, se trouvant dans le titre de l'œuvre, était difficilement contournable. L'auteur fournissait pourtant dans le corps du texte des indications précises sur la nature dialectale et sur le sens du terme wuthering, mais il semble pourtant que le français ne pouvait accueillir ce sens de façon simple et univoque ${ }^{38}$.

Les termes francisés ferroniens posent un problème de traduction d'un ordre nettement plus complexe et permettaient en théorie une quatrième stratégie de la part des traducteurs: une réexpression équivalente par la formation d'un terme où soient confrontés l'anglais et une langue autre que le français ${ }^{39}$. Trouver une seconde langue à combiner avec l'anglais ne pose pas problème. Ce qui en pose cependant un, et insurmontable, c'est de trouver pour l'anglais une langue de combinaison avec laquelle elle soit dans le même rapport identitaire qu'elle ne l'est avec le français du Québec. Ces termes ferroniens procèdent du verbe et sont une variable qui relève indéniablement du niveau stylistique; mais elles sont aussi le terminus langagier d'une conjoncture historico-politique qui est unique, qui n'a d'équivalent exact nulle part ailleurs. De ce fait, ils partagent également une caractéristique fondamentale avec les phénomènes d'ordre culturel et idéologique dont une grande part de la détermination est extralangagière.

Les termes francisés de Jacques Ferron représentent une épreuve ultime de l'étranger; une frontière du traductible dans le passage de laquelle le traducteur ne 
peut jamais être tout à fait en règle dans son périple vers la reconfiguration du texte d'origine en langue anglaise.

\section{NOTES}

1. Julien Bigras et Jacques Ferron, Le désarroi. Correspondance entre Julien Bigras et Jacques Ferron, Montréal, VLB Éditeur, 1988, p. 43.

2. Ginette Michaud, (dir.) avec la collaboration de Patrick Poirier, L'autre Ferron, Montréal, FidesCÉTUQ, coll. «Nouvelles études québécoises», 1995.

3. Jacques Ferron, L’amélanchier, Montréal, Éditions du Jour, 1970 / Montréal, Typo, 1992.

4. Jacques Ferron, Les roses sauvages (petit roman suivi d'une lettre d'amour soigneusement présentée), Montréal, Éditions du Jour, 1971 / Montréal, VLB Éditeur, 1990.

5. Jacques Ferron, Le salut de l'Irlande, Montréal, Éditions du Jour, 1970 / Outremont, Lanctôt Éditeur, coll. «Petite Collection Lanctôt», 1997.

6. Jacques Ferron, Le ciel de Québec, Montréal, Éditions du Jour, 1969 / Montréal, VLB Éditeur, 1979 / édition préparée par Pierre Cantin, avec la collaboration de Marie Ferron et de Gaëtane Voyer, Outremont, Lanctôt Éditeur, 1999.

7. Jacques Ferron, Les confitures de coings (suivi de Appendice aux confitures de coings ou Le congédiement de Frank Archibald Campbell), Montréal, Parti Pris, 1972 / Montréal, Typo, 1990.

8. Frank Scott était professeur de droit à l'Université McGill, et a d'ailleurs été doyen de la Faculté de droit de cette institution. Jacques Ferron et lui se sont côtoyés pendant plusieurs années au sein de différents partis politiques socialistes et socio-démocrates (CCF, PSD, NPD) et ont longtemps entretenu des rapports assez harmonieux. Le «congédiement» de l'œuvre de Ferron dont il est ici question est corollaire de la détérioration des rapports survenue entre les deux hommes dans la foulée des événements d'octobre 1970, où Frank Scott a pris parti pour Pierre Elliott Trudeau et l'occupation du Québec par l'armée canadienne, ce que Jacques Ferron ne lui a jamais pardonné par la suite: "[...]il a compris à un âge avancé que, membre d'une minorité dominante, c'était outrager le sens commun que de se prétendre homme de gauche, et qu'à l'instar de tous ces Messieurs de la McGill University il était au fond un Rhodésien et qu'en faisant de son mieux il pouvait tout au plus atteindre le centre droit, dans l'éventail des partis.» (Jacques Ferron, Escarmouches. La longue passe, volumes 1 et 2, Montréal, Leméac, 1975, volume 1, p. 135).

9. Les nombreux textes écrits par Ferron concernant cette question ont été réunis dans un recueil intitulé: Le contentieux de l'Acadie, édition préparée par Pierre Cantin, Marie Ferron et Paul Lewis avec la collaboration de Pierre L'Hérault, Montréal, VLB Éditeur, 1991.

10. Jacques Ferron discute de cette question à maintes reprises dans son œuvre. Voir notamment Escarmouches. La longue passe (volume 1, p. 31-32 et 57; et volume 2, p. 169-170).

11. Voir notamment, Escarmouches. La longue passe (volume $1:$ «Le clin d'œil de Shakespeare», p. 95, et volume 2: «À chacun son Dieu», p. 198-201).

12. Concernant cet auteur, voir particulièrement l'étude de Betty Bednarski, Translating Ferron, Ferron Translating: Thoughts on an Example of "Translation within", Meta, vol. 45-1, p. 37-51, où est analysée en détail la traduction française d'un poème de Samuel Butler dans La nuit / Les confitures de coings de Jacques Ferron.

13. La position de Ferron concernant ce romancier américain, de même que l'opposition implicite exprimée par ses épithètes «anglifié» ou "anglaisé» sont discutées par Ginette Michaud, «Lire à l'anglaise», dans Ginette Michaud (réd.), avec la collaboration de Patrick Poirier, L'autre Ferron, p. 157.

14. Lise Gauvin Langagement. L'écrivain et la langue au Québec, Montréal, Éditions du Boréal, 2000 (voir principalement p. 23 à 32).

15. Richard Patry, «Stratégie d'appropriation du lexique étranger dans l'œuvre de Jacques Ferron: l'intrus mis à la marge», dans Yves Duhoux (dir.) Langue et langues. Hommage à Albert Maniet, Louvainla-Neuve, Peeters, 1998, (p. 197-223).

16. Selon les conventions terminologiques en vigueur dans le domaine de la lexicométrie, le terme «occurrence» renvoie au nombre total de mots relevés dans l'œuvre de l'auteur, alors que «vocable» indique le nombre de mots différents au sein de cet ensemble.

17. Il est important de souligner que le vocable Elséneur est associé de près à l'œuvre de Shakespeare, et qu'il nous ramène donc, indirectement, à l'alter ego anglais (anglophone) du Québec.

18. La catégorisation des œuvres de Ferron dans des genres textuels est loin d'être simple et soulève de nombreuses questions qui sont discutées en détail aux pages p. 208-210 de l'étude ici résumée. 
19. Cette dernière partie comportait l'examen de plusieurs sous-questions dont la présentation nécessiterait un développement étendu qui ne serait pas directement pertinent pour le propos de la présente étude.

20. Les termes francisés de Jacques Ferron constituent des apparitions furtives et malicieuses dans un cadre langagier où le français est parfaitement maitrisé. L'hétérogène linguistique n’a cependant pas toujours une mine aussi réjouie et peut parfois refléter une réalité autrement pathétique d'indigence culturelle et langagière. Sherry Simon, dans Le trafic des langues. Traduction et culture dans la littérature québécoise, Montréal, Éditions du Boréal, 1994, propose une étude intéressante de cette problématique dans un chapitre qui a pour titre «La langue blessée» (p. 111-127).

21. Pour un index complet des noms de lieux et de personnes dans l'œuvre de Jacques Ferron, voir Luc Gauvreau, Noms et encyclopédie dans l'œuvre de Jacques Ferron. Suivi d'un index onomastique général, tomes 1 et 2, Montréal, Mémoire de maîtrise du Département d'études françaises de l'Université de Montréal, 1994. Cet index peut également être consulté en ligne sur le site Internet réalisé par Luc Gauvreau et consacré à Jacques Ferron. L'adresse de ce site est [http://www.ecrivain.net/ferron/].

22. Il s'agit ici de Lester Bowles Pearson (1897-1972), homme politique canadien qui fut d'abord ministre fédéral des Affaires étrangères dans le gouvernement libéral de Louis S. Saint-Laurent de 1948 à 1957, année où il devint lui-même chef du Parti libéral fédéral. Il fut premier ministre du Canada de 1963 à 1967 pour ensuite se retirer de la vie politique.

23. Une bibliographie, la plus complète possible, des traductions de l'œuvre de Jacques Ferron est présentée en annexe à la présente étude.

24. Voir entre autres le très beau texte qu'elle a écrit en hommage à l'auteur lors de son décès: Betty Bednarski, «Rereading Ferron», Antigonish Review, no. 61, 1985, pp. 43-49.

25. Annette Hayward et André Lamontagne, «Le Canada anglais: une invention québécoise?», Voix et images, 72, 1999, p. 460-479. Les dix premiers auteurs les plus étudiés sont les suivants: Gabrielle Roy, Anne Hébert, Marie-Claire Blais, Michel Tremblay, Hubert Aquin, Saint-Denys Garneau, Jacques Ferron, Louis Hémon et Nicole Brossard (p. 474-475).

26. Pierre Daviault fut un traducteur de grande notoriété dont la préoccupation principale a toujours été l'amélioration de la qualité de la langue française au Canada. Il a été Surintendant du Bureau des traductions du gouvernement du Canada à partir de 1954. Il fut également professeur à l'École de traduction de l'Université d'Ottawa et a rédigé plusieurs ouvrages de traduction et de lexicographie monolingue et bilingue. Pierre Daviault a été membre de la Société royale du Canada, dont il a assumé la présidence en 1958-1959, et il a enfin été président de la Société des écrivains du Canada de 1958 à 1961.

27. Walter Benjamin, «L'abandon du traducteur. Prolégomènes à la traduction des "Tableaux parisiens" de Charles Baudelaire», traduction et notes de Laurent Lamy et Alexis Nouss, TTR, «L'essai sur la traduction de Walter Benjamin », traductions critiques, sous la direction d'Alexis Nouss, 10-2, 1997, p. 13-69.

28. Cette conception selon laquelle le récepteur ne doit pas être prise en considération dans l'évaluation d'une œuvre d'art est également affirmée très catégoriquement dans la Poétique d'Aristote: «Quant au spectacle, qui exerce la plus grande séduction, il est totalement étranger à l'art et n'a rien à voir avec la poétique» (50b/15-21, p. 57). Pour l'auteur, le ferment poétique de l'œuvre est dans l'œuvre elle-même, et il ajoute même que, concernant le spectacle, l'art du fabricant de décors a plus d'importance que celui du poète! (Roselyne Dupont-Roc et Jean Lallot, Aristote, La poétique. Le texte grec avec une traduction et des notes de lecture, Paris, Éditions du Seuil, 1980).

29. Pierre Deshusses, "Le traducteur est un écrivain», "Théorie et pratique de la traduction III. La traduction littéraire.» Cahiers internationaux du symbolisme, 92-93-94, 1999, p. 49-53.

30. Jean Delisle, L'analyse du discours comme méthode de traduction. Initiation à la traduction française de textes pragmatiques anglais, Ottawa, Éditions de l'Université d'Ottawa, 1980, p. 22.

31. Voir entre autres Jacky Martin, «Essai de redéfinition du concept de traduction », Meta, 27-4, 1982, p. 357-374, également l'étude de Raquel de Pedro, «The Translatability of Texts: A Historical Overview», Meta, 44-4, 1999, p. 546-559, enfin, celle de Betty Bednarski, «Translating Ferron, Ferron Translating: Thoughts on an Example of "Translation within" ", où la dimension interculturelle de la traduction est aussi abordée.

32. S'intègrent ici les 12 tendances déformantes identifiées par Antoine Berman dans son analytique de la traduction (Antoine Berman, «La traduction comme épreuve de l'étranger», Texte. Revue de critique et de théorie littéraire, numéro thématique "Traduction/Textualité», 1985, p. 67-82). Voir également en ce qui concerne la difficulté à traduire les expressions idiomatiques Pamela J. Olubunmi 
Smith, «Literary Translation and Culture Conciousness: The Experience of Translating D.O. Fagunwa's Igbo Olodumare from Yoruba into English», Meta, 38-2, 1993, p. 218-225.

33. Un bon exemple de manipulation idéologique dans la traduction du journal d'Anne Frank est présenté dans André Lefevere, Translation, Rewriting and the Manipulation of Literary Fame, London (UK), Routledge, coll. «Translation Studies», 1992, p. 68-70.

34. L'utilisation du gras et du soulignement dans les exemples qui suivent est le fait de notre intervention et a pour but de mettre en évidence les termes anglais francisés et la façon dont ils ont été traduits.

35. Betty Bednarski, Autour de Ferron. Littérature, traduction, altérité. Toronto, Éditions du GREF, coll. «Traduire, écrire, lire», 1989, p. 51-58. Et Betty Bednarski, "From ouèredéare? to Soçaurez: Translating the english of Jacques Ferron», dans Sherry Simon (dir.) Culture in Transit, Montréal, Véhicule Press, 1995, 110-133.

36. Katy Mezei, «Translations», University of Toronto Quarterly, 54-4, 1985, p. 382-399.

37. Ray Ellenwood s'est également expliqué de certains de ses choix traductionnels, mais concernant des phénomènes autres que celui considéré dans cette étude. Ray Ellenwood, «Les chians de la traduction, ou comment j'ai esquivé une meute de difficultés diaboliques en allant du Ciel de Québec vers The Penniless Redeemer», Littératures, 9-10, 1992, p.151-158. Et, Ray Ellenwood, "Somes notes on the politics of translation", Atkinson Review of Canadian Studies, 2-1, 1984, p. 25-28.

38. Concernant les différents titres en traduction française de cette œuvre d'Emily Brontë, voir l'excellente étude de Christine Klein-Lataud, «Nom propre et traduction », dans Martine Léonard et Élisabeth Nardout-Lafarge (dir.), Le texte et le nom, Montréal, XYZ Éditeur, coll. «Documents », 1996, p. 169-180.

39. En traduisant, par exemple, le terme ferronien «ouatchâte! " par une anglicisation rédactionnelle du terme allemand équivalent: «forzisht!» [Vorsicht!].

40. Dans le cas de cet ouvrage, le traducteur a choisi pour titre le nom d'un personnage qui arrive à la toute fin du roman, et qui est celui d'un enfant né dans le village des Chiquettes lors de la fondation de la paroisse de Sainte-Eulalie: Rédempteur Fauché. L'auteur termine d'ailleurs son récit en annonçant quel sera le titre de son prochain épisode — qu'il n'a malheureusement jamais écrit: «[...] tant il est vrai qu'on ne saurait écrire une chronique sans en annoncer la suite en même temps qu'on l'achève. Elle s'intitulera: La vie, la passion et 1a mort de Rédempteur Fauché» (p. 396). Ajoutons enfin, que cette dénomination évocatrice a été suggérée à Jacques Ferron par un fait divers concernant un individu du nom de «Rédempteur Faucher» qui faisait partie du monde du petit crime organisé de la région de Québec et qui serait mort de façon suspecte au milieu des années 1960.

\section{ANNEXE}

\section{Livres de Jacques Ferron traduits}

(1972) Tales from the Uncertain Country, Toronto, Anansi, 101 p. Traduction de Betty Bednarski. (Jacques Ferron, Contes du pays incertain, Éditions d'Orphée, 1962. Voir également, Jacques Ferron, Conte (Contes anglais, Contes du pays incertain, Contes inédits), Montréal, Hurtubise, HMH, 1968 (rééditions en 1976 et 1985) / Montréal, Bibliothèque québécoise, 1993 (réédition en 1997) / édition critique préparée par Jean-Marcel Paquette, Montréal, Presses de l'Université de Montréal, coll. «Bibliothèque du Nouveau Monde», 1998).

(1974) Dr. Cotnoir, A Novel by Jacques Ferron, Montreal, Harvest House, 86 p. Traduction de Pierre Cloutier. (Jacques Ferron, Cotnoir, Montréal, Éditions d'Orphée, 1962 / Montréal, Éditions du Jour, 1970 / Montréal, VLB Éditeur, 1981.)

(1975) The Juneberry Tree, A Novel by Jacques Ferron, Montreal, Harvest House, 157 p. Traduction de Raymond Y. Chamberlain. (Jacques Ferron, L’amélanchier, Montréal, Éditions du Jour, 1970 / Montréal, Typo, 1992.)

(1975) The Saint Elias, A Novel by Jacques Ferron, Montreal, Harvest House, 145 p. Traduction de Pierre Cloutier. (Jacques Ferron, Le Saint-Élias, Montréal, Éditions du Jour, 1972 / Montréal, Typo, 1993.)

(1976) Wild Roses, followed by a Love Letter, Toronto, McClelland and Stewart, 123 p. Traduction de Betty Bednarski. (Jacques Ferron, Les roses sauvages, suivi d'une lettre d'amour soigneusement présentée), Montréal, Éditions du Jour, 1971 / Montréal, VLB Éditeur, 1990.) 
(1977) Quince Jam (Papa Boss, Quince Jam, Credit due, Appendix to Quince Jam or the Sacking of Frank Archibald Campbell), Toronto, Coach House Press, «Coach House Quebec Translations », 262 p. Traduction de Ray Ellenwood. (Jacques Ferron, Papa boss, suivi de La créance, Montréal, Parti Pris 1972 / Montréal, Typo 1990; Jacques Ferron, Les confitures de coings, suivi du Congédiement de Frank Archibald Cambell, Montréal, Parti Pris, 1972 / Montréal, Typo, 1990.)

(1980) The Cart. A Novel by Jacques Ferron, Toronto, Exile Editions, 144 p. Traduction de Ray Ellenwood. (Jacques Ferron, La charrette, Montréal, Hurtubise HMH, 1968 / Montréal, Typo, 1994.)

(1984) Selected Tales of Jacques Ferron, Toronto, Anansi, 245 p. Traduction de Betty Bednarski. (Jacques Ferron, Contes, (Contes anglais, Contes du pays incertain, Contes inédits), Montréal, Hurtubise, HMH, 1968 (rééditions en 1976 et 1985) / Montréal, Bibliothèque québécoise, 1993 (réédition en 1997) / édition critique préparée par Jean-Marcel Paquette, Montréal, Presses de l'Université de Montréal, coll. «Bibliothèque du Nouveau Monde», 1998.)

(1984) The Flowering Suns, mémoire de maitrise, Toronto, York University, 210 p. Traduction de Julie May Stockton. (Jacques Ferron, Les grands soleils, dans Théâtre 1. L'ogre, Tante Élise ou le prix de l'amour, Cazou ou le prix de la virginité, Le Don Juan Chrétien, Les grands soleils, Montréal, Typo, 1990.)

(1984) The Penniless Redeemer, Toronto, Exile Editions, 342 p. Traduction de Ray Ellenwood. (Jacques Ferron, Le ciel de Québec, Éditions du Jour, 1969 / Montréal, VLB Éditeur, 1979 / édition préparée par Pierre Cantin avec la collaboration de Marie Ferron et de Gaëtane Voyer, Outremont, Lanctôt Éditeur, 1999. ${ }^{40}$ )

\section{Périodiques}

Une livraison de la revue Brick ( $\mathrm{n}^{\circ} 16$, Automne 1982) a été entièrement consacrée à la diffusion de traductions de textes de Jacques Ferron. Le contenu de ce numéro de la revue est le suivant:

«The Jailer's Son», p. 6-7, traduction de Betty Bednarski. (Jacques Ferron, «Le fils du geôlier», dans Contes, p. 211-216.)

«The Parakeet», pp. 7-8, traduction de Betty Bednarski. (Jacques Ferron, «La perruche», dans Contes, p. 158-160.)

«Servitude», p.8, traduction de Betty Bednarski. (Jacques Ferron, «Servitude», dans Contes, p. 20-22.)

«François Hertel's Beard», p. 9-14, traduction de Ray Y. Chamberlain. (Jacques Ferron, La barbe de François Hertel suivi de Le licou, Montréal, Éditions d'Orphée, 1956 / Montréal, VLB Éditeur 1981.)

«Dollard des Ormeaux, Three Times Dead», pp. 14-17, traduction de Elisabeth A. Darling. (Jacques Ferron, «Sieur Dollard, trois fois mort», dans Historiettes, Montréal, Éditions du Jour, 1969, p. 135-144.)

"All is not Lost», p. 17, traduction de Ray Ellenwood. (Jacques Ferron, "Tout n'est pas perdu ", dans Historiettes, Montréal, Éditions du Jour, 1969, p. 104-106.)

«God and his Scribes», p. 18-19, traduction de Ray Ellenwood. (Jacques Ferron, «Dieu et ses scribes », dans Du fond de mon arrière-cuisine, Montréal, Éditions du Jour, 1973, p. 149-154.)

«The Dragon, the Maiden and the Child», pp. 19-20, traduction de Ray Ellenwood. (Jacques Ferron, «Le dragon, la pucelle et l'enfant», dans Escarmouches. La longue passe, tome 1, Montréal, Leméac, 1975, p. 125-128.)

"One Death too Many », pp. 21-25, traduction de Ray Ellenwood. (Jacques Ferron, "Une mort de trop », dans Escarmouches. La longue passe, tome 1, Montréal, Leméac, 1975. p. 103-118.)

«Purple Loosestrife», pp. 25-30, traduction de Ray Ellenwood. (Jacques Ferron, «Les salicaires», dans $D u$ fond de mon arrière-cuisine, Montréal, Éditions du Jour, 1973, p. 267-280.)

«Hemp: Its arrival», pp. 31-32, traduction de Raymond Y. Chamberlain. (Jacques Ferron, «Le chanvre, son arrivée», dans $D u$ fond de mon arrière-cuisine, Montréal, Éditions du Jour, 1973, p. 71-75.) 
«Its Dialectic», pp. 32-33, traduction de Raymond Y. Chamberlain. (Jacques Ferron, «Sa dialectique», dans Du fond de mon arrière-cuisine, Montréal, Éditions du Jour, 1973, p. 75-78.)

«The Virtue of Agnus Castus», pp. 33-34, traduction de Raymond Y. Chamberlain. (Jacques Ferron «La vertu de l'Agnus-Castus», dans L'Information médicale et paramédicale, 28- 9, 16 mars 1976, p. 14.)

«The Pantagruelion I», pp. 34-35, traduction de Raymond Y. Chamberlain. (Jacques Ferron, «Le Pantagruelion I», dans L'Information médicale et paramédicale, 29-21, 20 septembre 1977, p. 7.)

«The Pantagruelion II», p. 35, traduction de Raymond Y. Chamberlain. (Jacques Ferron, "Le Pantagruelion II", dans L'Information médicale et paramédicale, 29-22, 4 octobre 1977, p.25.

«La Sorgne», p. 36-39, traduction de Ray Ellenwood. (Jacques Ferron, «La sorgne», dans Julien Bigras et Jacques Ferron, Le désarroi. Correpondance entre Julien Bigras et Jacques Ferron, Montréal, VLB Éditeur, 1988, p. 121-132.)

"The Alias of the "NO" and of Nothingness", pp. 40-41, traduction de Raymond Y. Chamberlain. (Jacques Ferron, «L'alias du non et du néant», dans Le Devoir, 19 avril 1980, p. 21.)

"The Execution of Maski», pp. 41-46, traduction de Ray Ellenwood. (Jacques Ferron, «L'exécution de Maski» dans Rosaire. Précédé de l'exécution de Maski, Montréal, VLB Éditeur, 1981, p. 11-36.)

\section{Textes épars}

"The Pigeon and the Parakeet», traduction de Betty Bednarski, dans The Canadian Fiction Magazine, 15, 1974, pp. 14-16. (Jacques Ferron, «Le pigeon et la perruche», dans Contes, p. 228-232.)

"Claude Gauvreau», traduction de Ray Ellenwood, dans Exile, A Journal of Canadian and International Writing, 3-2, 1976, pp. 21-57. (Jacques Ferron, «Claude Gauvreau», dans Du fond de mon arrière-cuisine, Montréal, Éditions du Jour, 1973 p. 202-265.)

«The Old Heathen», traduction de Betty Bednarski, Exile, A Journal of Canadian and International Writing, 3-2, 1976, pp. 14-15. (Jacques Ferron, «Le vieux payen», dans Contes, p. 202204.)

"The Wool Nightshirt and the Horsehair Tunic», traduction de Betty Bednarski, Exile, A Journal of Canadian and International Writing, 3-2, 1976, pp. 11-13. (Jacques Ferron, «La laine et le crin», dans Contes, p. 195-198.)

«The Parrot», traduction de Betty Bednarski, Exile, A Journal of Canadian and International Writing, 3-2, 1976, pp. 16-19. (Jacques Ferron, «Le perroquet», dans Contes, p. 71-77.)

«La sorgne», traduction de Ray Ellenwood, dans Exile, A Journal of Canadian and International Writing, 5-3/4, 1978, pp. 78-86. (Jacques Ferron, «La sorgne», dans Julien Bigras et Jacques Ferron, Le désarroi. Correpondance entre Julien Bigras et Jacques Ferron, Montréal, VLB Éditeur, 1988, p. 121-132.)

"The Parrot», traduction de Betty Bednarski, dans Short Short Stories, Toronto, Holt, Rinehart and Winston, 1981, pp. 7-10. (Jacques Ferron, «Le perroquet», dans Contes, p. 71-77.)

«The Bridge», traduction de Betty Bednarski, dans Introduction to Fiction, Toronto, Holt, Rinehart and Winston, 1982, p. 10. (Jacques Ferron, «Le pont», dans Contes, p. 68-70.)

«The Chronicle of L'Anse Saint-Roch», traduction de Wayne Grady, dans Wayne Grady (dir.) The Penguin Book of Modern Canadian Short Stories, New York, Penguin Books, 1982, pp. 255-265. (Jacques Ferron, «Chronique de l'Anse Saint-Roch, dans Contes, p. 263-276.)

«Mélie and the Bull», traduction de Betty Bednarski, dans Richard Teleky (dir.) The Oxford Book of French-Canadian Short Stories, Oxford (UK), Oxford University Press, 1983, pp. 180-196. (Jacques Ferron, «Mélie et le bœuf», dans Contes, p. 37-55.)

«The Chichemayais», traduction de Betty Bednarski, dans Ethos, 1-2, 1983, pp. 10-14. (Jacques Ferron, "Le chichemayais», dans La conférence inachevée. Le pas de Gamelin et autres récits, édition préparée par Pierre Cantin, Marie Ferron et Paul Lewis, Montréal, VLB Éditeur, 1987, p. 95-107/ édition préparée par Pierre Cantin et Marcel Olscamp, Outremont, Lanctôt Éditeur, coll. «Petite Collection Lanctôt», 1998, p. 101-114.) 
"Chronicle of Anse Saint-Roch », traduction de Betty Bednarski, dans The Antigonish Review, 56, 1984, pp. 55-65. (Jacques Ferron, "Chronique de l'Anse Saint-Roch, dans Contes, p. 263276.)

«Martine Continued», traduction de Larry Shouldice, dans W. H. New (dir.) Canadian Fiction. From Myth to Modern, Scarborough (Ontario), Prentice-Hall Canada, 1986, pp. 224-229. (Jacques Ferron, «Suite à Martine», dans Contes, p. 173-182.)

"The Dead Cow in the Canyon », traduction de Betty Bednarski, dans G. Hancock (dir.) Invisible fictions. Contemporary Stories from Quebec, Toronto, Anansi, 1987, pp. 359-388. (Jacques Ferron, «La vache morte du canyon», dans Contes, p. 101-135.)

"Chronicle of Anse Saint-Roch», traduction de Betty Bednarski, dans From Ink Lake: Canadian Stories, Toronto, Lester and Orpen Dennys, 1990, pp. 115-125. (Jacques Ferron, «Chronique de l'Anse Saint-Roch, dans Contes, p. 263-276.)

"The Provinces», traduction de Betty Bednarski, dans J. R. Struthers (dir.) The Possibilities of Story, volume 1, Toronto, McGraw-Hill Ryerson Limited, 1991, pp. 106-109. (Jacques Ferron, «Les provinces», dans Contes, p. 84-89.)

"The Jailer's Son », traduction de Betty Bednarski, dans Short Fiction: An Introduction Anthology, Toronto, Harcourt, Brace and Jovanovich, 1992, pp. 894-897. (Jacques Ferron, «Le fils du geôlier», dans Contes, p. 211-216.)

"Chronicle of Anse Saint-Roch», traduction de Betty Bednarski, dans D. Lampe (dir.) Myth and Voices: Contemporary Canadian Fiction, New York, White Pine Press, 1993, pp. 162-175. (Jacques Ferron, «Chronique de l'Anse Saint-Roch», dans Contes, p. 263-276.)

«Chronicle of Anse Saint-Roch», traduction de Betty Bednarski, dans M. Cohen et W. Grady (dir.) The Quebec Anthology, 1830-1990, Ottawa, University of Ottawa Press, 1996. (Jacques Ferron, «Chronique de l'Anse Saint-Roch », dans Contes, p. 263-276.) 